Itinéraires Itinéraires

Littérature, textes, cultures

2010-2 | 2010

Les blogs

\title{
Ce que les réseaux font à la littérature
}

Réseaux sociaux, microblogging et création

\section{Alexandre Gefen}

\section{OpenEdition}

Journals

Édition électronique

URL : http://journals.openedition.org/itineraires/2065

DOI : $10.4000 /$ itineraires.2065

ISSN : 2427-920X

Éditeur

Pléiade

\section{Édition imprimée}

Date de publication : 1 juillet 2010

Pagination : 155-166

ISBN : 978-2-296-12012-9

ISSN : 2100-1340

Référence électronique

Alexandre Gefen, «Ce que les réseaux font à la littérature », Itinéraires [En ligne], 2010-2 | 2010, mis en ligne le 01 juillet 2010, consulté le 10 décembre 2020. URL : http://journals.openedition.org/itineraires/ 2065 ; DOI : https://doi.org/10.4000/itineraires.2065

\section{(c) (i) (3)}

Itinéraires est mis à disposition selon les termes de la licence Creative Commons Attribution - Pas d'Utilisation Commerciale - Pas de Modification 4.0 International. 


\title{
Ce que les réseaux font à la littérature. Réseaux sociaux, microblogging et création ${ }^{1}$
}

\begin{abstract}
Microblogging - a brief, flowing, asynchronous writing of oneself-has not been a long time making Facebook or Twitter places for a literary writing. Diverting their device, it leads to forms submitted to experimental constraints. Better still, the fragmented writing, with its universe of affective flow awaiting to be echoed, goes together with the numerical conversion of human experience : far from being the empire of triteness and ephemeralness, microblogging induces literature to quit the fields thought for literary expression and intrudes itself on the inside of social relationships.
\end{abstract}

Keywords : Twitter, Facebook, social networks, numerical writings, microblogging Mots clés : Twitter, Facebook, réseaux sociaux, écritures numériques, microblogging

Le cortège passait et j'y cherchais mon corps. Apollinaire, Cortège

Il existe un nombre non négligeable d'études consacrées à la pratique des blogs - considérés dans la perspective de la sociologie, de la psychologie sociale ou de l'anthropologie culturelle ${ }^{2}$ - mais, que les blogs soient pensés comme un espace de mise en scène, de médiation, d'expression ou de construction identitaire, leur statut esthétique est en général rapporté par analogie à celui du journal personnel et rattaché au modèle discursif des écritures de vies. Cette

1. Mercià@ck_amontour,@fbon,@reneaudet,@marind,@liminaire,@piotrr70,@calimaq, @frederiClement, @Alice_, @remuenet et tant d'autres pour leurs remarques.

2. Notamment les travaux de Serge Tisseron, L'Intimité surexposée, Paris, Ramsay, 2001 ou l'essai "Cher écran...» de Philippe Lejeune, Paris, Seuil, 2001; on lira également l'article intéressant de Janique Laudouar : « Désir de s'exposer, désir d'écrire », Écritures hypertextuelles, http://www.cndp.fr/archivage/valid/54656/54656-7748-7732.pdf; pour une bibliographie à jour, on consultera le site de Philippe Lejeune : http://www.autopacte.org/Etudes_recentes.html. 
supposée forme simple, méta ou supragénérique est alors située en dehors d'une tradition normative ou intertextuelle consacrée, et reléguée aux frontières extérieures du champ littéraire. Bloguer serait d'abord un acte social, directement ou indirectement performatif qui, de fait, ne s'inscrit que difficilement dans les critères définitoires de la « littérature littéraire » : faiblement contractualisée et possédant sa sphère référentielle propre, l'écriture par blog résiste à l'opposition fait/fiction (critère de fictionnalité) qui pourrait la faire admettre dans le corpus littéraire traditionnel; formalisée par réaction à des contraintes technologiques exogènes, elle peine à opérer cette ostentation du signifiant et cette dénudation des procédés qui la qualifierait de littéraire par diction. Ainsi, rares sont les études ayant fait du blog un genre littéraire en soi (c'est-à-dire, et quelle que soit la définition du genre que l'on retienne, une forme matrice de sens), y compris dans le monde critique anglo-saxon, pourtant ouvert à une théorie large des médias et attentif au pouvoir configurant des supports textuels. La pratique du microblogging serait située en amont de l'œuvre dont il constituerait au mieux l'expérience ou l'atelier (chez François Bon, qui réunit dans Tumulte les fragments résultant d'une « écriture quotidienne, non préméditée, réalisée directement sur serveur Internet, du $1^{\text {er }}$ mai 2005 au 11 mai $2006^{3} \gg$ ) ou en aval, comme un dispositif d'escorte complétant et enrichissant le projet organique de l'entreprise littéraire en lui annexant une hypostase mobile et collaborative, mais, comme œuvre, elle ne correspondrait pas à cette forme totale rêvée par ceux qui ont vu, depuis Julio Cortázar, dans l'écriture hypertextuelle et combinatoire un possible Grand Livre couronnant notre histoire littéraire.

Cette difficulté d'analyse et cette incompatibilité avec les catégories natives de littérarité issues de la philosophie kantienne du beau, philosophie de l'art ne discriminant pas, comme l'a montré Jean-Marie Schaeffer, évaluation et définition ${ }^{4}$, et renvoyant mécaniquement les productions numériques au rang de publications de circonstances infraculturelles, s'accroissent encore si nous réfléchissons à cette forme si originale qu'est le microblogging. On peut définir celle-ci comme une écriture de soi brève (limitée à cent quarante caractères sur Twitter), en flux (c'est-à-dire insérée dans un continuum à la fois horizontal et vertical d'autres microtextes), asynchrone et donc en pointillé (à la différence des dispositifs conversationnels offerts par les messageries instantanées), telle que des outils sociaux comme la plate-forme sociale intégrée Facebook, l'agrégateur Friendfeed, l'outil de discussion en réseau Twitter ou son alternative ouverte Identi.ca, la produisent ${ }^{5}$. À la différence du blog par fragments brefs dont on peut les rapprocher (je pense à la pratique d'Éric

3. Arnaud Maïsetti, «Internet : de la condition du blogueur », Carnets, 23 février 2009, http://arnaudmaisetti.net/spip/spip.php?article10, consulté le 19 avril 2010.

4. Voir Jean-Marie Schaeffer, L'Art de l'âge moderne, Paris, Gallimard, 1992.

5. Il existe quantité d'autres outils de microblogging: Tumblr, Plurk par exemple, mais l'économie du Net semble bien avoir fait, à l'heure où j'écris ces lignes, son choix, que viendra peut-être renverser Google Wave. 
Chevillard dans « L'autofictif $^{6}$ », énigmatique chronique intérieure en forme de notules poético-ironiques journalières), ces pratiques de microblogging se caractérisent par plusieurs spécificités fortes. En premier lieu, elles relèvent d'une écriture par « statuts », détournant un dispositif de liens faibles pensé pour le maintien a minima de relations sociales dans une communauté et destiné à assurer la continuité du groupe dans sa mobilité territoriale. Elles s'inscrivent dans une sorte de mi-chemin entre le public et le privé, l'informationnel et le communicationnel, dans une forme de communication par broadcast qui n'est ni la publication éditoriale classique, ni sa modalité revuistique, ni le dialogue, ni la conférence : les messages émis par Twitter sont publics, ce qui les conduit souvent à être savamment maîtrisés, mais noyés dans une masse que seuls un travail de regroupement volontaire par mots clés (hashtags) ou l'œuvre hasardeuse de la sérendipité ${ }^{7}$ peuvent démêler; ceux de Facebook s'adressent à une communauté lectorale choisie et resserrée, mais conservent toute fluidité en ne visant pas de destinataire précis ${ }^{8}:$ ils rencontrent donc une communauté interprétative à la fois locale et mondiale, un sens à la fois public et privé, un auditoire présent en temps réel et à venir par archivage et indexation, toutes formes d'articulation de la parole et de sa réception éminemment originales dans notre histoire culturelle. Dans ses modalités énonciatives, l'écriture par Twitter relève d'un détournement d'une technologie au profit d'un désir d'écriture : celui de produire une théorie d'états d'âme, une météorologie de l'humeur et du lieu, un flux atomistique d'autant plus transitoire qu'il accepte de dissoudre sa propre voix dans le bruit immense de la présence textuelle numérique d'autrui. Cette discontinuité, qui interdit de constituer le texte en une nappe unifiée dont la lecture serait prévisible et maîtrisable, produit des fragments qui s'exposent et se détachent poétiquement de la temporalité énonciative globale de la timeline sociale pour acquérir une portée expressive. C'est aussi que les gazouillages des tweets et autres statuts sont indissociables des rétroactions, gloses, expansions poétiques, croisements qu'ils engendrent, du « tumulte » du

6. http://1-autofictif.over-blog.com/. Les 328 premiers billets du blog, publiés entre le 18 septembre 2007 et le 17 septembre 2008, sont parus avec le sous-titre Journal : Éric Chevillard, L'Autofictif. Journal 2007-2008, Paris, Éditions de l'Arbre vengeur, 2009.

7. Il existe un mode d'accès à Twitter permettant l'entrée par découpage chronologique, en quelque sorte au hasard parmi les millions de messages échangés quotidiennement; quant à Facebook, et ses 300 millions d'utilisateurs, il constitue un web dans le Web, à peine équipé d'outils de rediffusion des messages d'autrui à la Twitter, où le signalement contrôlé d'une parole individuelle est éminemment problématique.

8. Pour les créateurs de Twitter, ce réseau n'est au demeurant pas conçu comme un réseau de diffusion mais avant tout comme un espace d'interaction, et l'usage de plus en plus commun de Twitter pour une diffusion centrifuge d'annonces et de publicités en dehors d'un cercle de destinataires est une extension imprévue du projet, dont le principal moteur de succès est, comme beaucoup d'outils Internet, un polymorphisme qui ne présume pas de l'ensemble des usages qui peut en être fait. Sur l'analyse de la sociologie communicationnelle de Twitter, on pourra consulter : Palpitt, « Une approche sociologique de Twitter », ReadWriteWeb France, 10 septembre 2009, http://fr.readwriteweb. com/2009/09/10/analyse/une-approche-sociologique-de-twitter, consulté le 19 avril 2010. 
corps social qu'ils recherchent, pour employer une expression de François Bon : leur poéticité est celle de nœuds, d'interstices, de brouillages par interférence, où la littérature se crée par une autre forme d'universalisation du privé que celle, supposément maîtrisée, à laquelle nous sommes habitués.

\section{Une littérature à contrainte}

La littérature investit les dispositifs techniques variés qu'elle rencontre, tendance renforcée par le goût moderne du formalisme, qui a vu avec les différentes formes de littérature à contraintes et de littérature à programme les écrivains inventer leurs propres servitudes. Ainsi le défi présenté par le microblogging, dont l'extrême brièveté est aux antipodes de la tradition livresque du grand roman, a été l'occasion de productions ayant pour point commun un jeu avec la brièveté et la réhabilitation ${ }^{9}$ d'une tradition d'écriture lapidaire ou fragmentaire ayant fourni dans le passé d'innombrables formes, aussi bien poétiques que réflexives ou narratives, de la maxime au haïku, de la «microfiction» à la Régis Jauffret aux «nouvelles en trois lignes» à la Félix Fénéon (dont les récits ont d'ailleurs été « twitterisés $\left.{ }^{10} »\right)$.

Je serais enclin à distinguer néanmoins deux types de pratiques : celle consistant à imposer une contrainte de lecture d'un récit produit sans appui sur son support et celle consistant à produire le texte dans l'espace même de l'outil de microblogging. On trouvera un bel exemple d'une telle opposition entre Le Paradisier de Frédéric Clément (auteur illustrateur de Magasin Zinzin, de Muséum et des Songes de la Belle au Bois dormant notamment) sous-titré « roman flottant distillé par Twitter ${ }^{11} »$, dont le rythme est donné par le croisement de textes sur Twitter (rediffusés sur Facebook) et d'extraits sonores donnés sur le site de partage musical Myspace, et Bashô, recueil poétique écrit par Françoise Kérisel et illustré par Frédéric Clément, à paraître chez Albin Michel, dont des extraits sont donnés sur Twitter en seconde intention et à des fins publicitaires.

Le cas des formes brèves inventées ou réinventées dans l'espace même des outils de publication numérique est sans doute quelque peu différent. J'ai déjà évoqué le blog «L'autofictif» d'Éric Chevillard qui, loin de se contenter des ressources désormais communes de l'autofiction, use du caractère séquencé de sa publication pour instaurer un désordre quotidien, une discontinuité stylistique et humorale dans le continuum de la page, et produit une théorie d'incongruités dont l'incidence est d'autant plus directe que les notules produites par Éric Chevillard peuvent être intégrées par un fil de syndication RSS aux plates-formes agrégées par lesquelles notre accès à l'actualité d'Internet se fait de plus en plus souvent (Google Reader, Netvibes) ou directement dans les navigateurs web ou les clients email proposés par

9. Sur ce statut problématique du fragment dans l'histoire littéraire, on relira le bel essai de Pascal Quignard, Une Gêne technique à l'égard des fragments, Paris, Fata Morgana, 1986.

10. Voir http://twitter.com/novelsin3lines.

11. http://leparadisier.blogspirit.com. 
plusieurs systèmes d'exploitation, et peuvent ainsi s'insérer dans notre quotidien. Prenons, une fois de plus au hasard, l'exemple du fragment 682 publié le dimanche 4 octobre 2009 :

C'est aussi simple que cela : dès que quelqu'un émet une proposition, le monde se scinde en deux camps : ses partisans et ses adversaires (mais j'entends déjà que des voix le contestent).

Au réveil, la paupière du bœuf se soulève sur l'œuf du jour.

Avec empressement, j'ai saisi la perche qu'elle me tendait. C'était le manche du râteau.

L'écriture de l'absurde, le détournement comique de l'aphorisme sérieux, la parodie de verset poétique visionnaire se renouvellent dans l'interlocution et le rythme que leur confèrent leur publication quotidienne et l'éthos simple et direct propre au blog. Même si une partie des textes d'Éric Chevillard a connu une publication en livre, les logiques insoumises et incoercibles du langage trouve dans l'écriture non totalisable d'une page, infinie, car quotidiennement modifiée - et en quelque sorte quotidiennement rejouée -, une forme idéale.

La contrainte de brièveté que s'impose Éric Chevillard n'est en rien un impératif technique, à la différence de l'écriture sur Twitter, libre de son rythme, mais cantonnée à cent quarante caractères. La production y invente des dérivés de formes traditionnelles, comme le Twaïk ${ }^{12}$ en poésie ou le Twiller en matière de roman - je passe sous silence d'autres formes comme celle du microconte ou de la minipoésie érotique ${ }^{13}$, ou encore l'étonnante proposition d'écriture du livret d'un opéra interactif sur Twitter lancée par le Royal Opera House ${ }^{14}$ ou celle d'un audiobook de cadavres exquis ${ }^{15}$. Le genre du Twiller a été inventé en 2008 par Matt Ritchel ${ }^{16}$, journaliste au New York Times qui justifie son dispositif (inspiré du film de Christopher Nolan, Memento) en imaginant un personnage amnésique qui reprend conscience isolé dans le désert avec, comme seul moyen de communication pour raconter et reconstruire son histoire, un smartphone avec accès à Twitter. La forme prend vite son autonomie, trouve son succès ${ }^{17}$, et se trouve reprise par des auteurs français attirés par

12. Voir notamment le site Small Poems (http://smallpoems.sbpoet.net/), l'inventaire de Wikipedia des différentes formes de Haïku numériques (http://en.wikipedia.org/wiki/Haiku\#Internet) et l'inventaire du Twitter Fan Wiki : http://twitter.pbworks.com/Twaiku-(haiku-written-in-twitter). 13. Voir l'inventaire de Francis Pisani : «Twiteromans », Transnets, 3 septembre 2008, http:// pisani.blog.lemonde.fr/2008/09/03/twiteromans, consulté le 19 avril 2010.

14. http://royaloperahouse.wordpress.com.

15. Voir http://www.bbcaudiobooksamerica.com/TradeHome/Blog/tabid/58/articleType/ ArticleView/articleId/110/Twitter-an-Audio-Story-with-Neil-Gaiman.aspx et le commentaire d'Actualitté : Clément S., "Neil Gaiman supervise un audiobook de cadavres exquis », 13 octobre 2009, http://www.actualitte.com/actualite/14166-Twitter-Neil-Gaiman-audiobookcadavre.htm, consulté le 19 avril 2010.

16. Matt Ritchel, «Introducing the Twiller », Bits, 29 août 2008, http://bits.blogs.nytimes. com/2008/08/29/introducing-the-twiller, consulté le 19 avril 2010.

17. Clément S., "Une première histoire écrite sur Twitter adaptée au cinéma », Actualitté, 
la tradition oulipienne et en particulier le néo-auteur Thierry Crouzet et son roman de gare, présenté comme dépourvu d'ambition littéraire, Croisade ${ }^{18}$, ou le roman écrit par LeRoy K. May et Éric Bourbonnais, Buboneka ${ }^{19}$. On retrouvera également sur Twitter des formes variées de romans-feuilletons usant de la publication différée pour créer du suspens : Thomas Drimm de Didier van Cauwelaert ${ }^{20}$ ou encore Le Roman d'Arnaud, qui se veut un « roman 2.0 » en reprenant le sobriquet par lequel le Web social se trouve souvent affublé et propose le dispositif suivant :

Trois auteurs, Gwen Catala, Christophe Sanchez et moi-même, blogueurs impénitents, auteurs convaincus que la lecture numérique va nous inciter à créer de nouvelles façons de raconter des histoires, vont se relayer pendant 40 jours et 40 nuits pour écrire un roman sur une page de fan Facebook et sur Twitter. 6 à 8 fois par jour, à des heures régulières, les statuts de la page du Roman d'Arnaud vont être mis à jour. Chaque auteur disposera de 420 caractères par statut pour faire évoluer l'histoire. Les lecteurs/fans pourront soit lire à heure fixe les mises à jour ou lire tout d'un seul trait à la fin de la journée. Ils pourront faire évoluer l'histoire en inscrivant des commentaires. Nous vous réservons d'autres surprises tout au long de cette expérience numérique ${ }^{21}$.

Quoi qu'on pense de l'ambition du « roman $2.0 »$ à modifier des pratiques de lectures pluriséculaires et à répondre à nos besoins de narration, toutes ces expérimentations, parfois proches des installations artistiques de l'art contemporain, se distinguent par le degré d'interaction qu'elles ménagent avec leurs utilisateurs, qui peut aller d'une publication signée et contrôlée à une écriture à plusieurs voix, en passant par le dialogue avec ceux que Frédéric Clément nomme ses « souffleurs » ou encore l'insertion conditionnelle du discours d'autrui après un processus de vote. Selon leurs protocoles d'interactivité, elles se différencient d'après leur degré de linéarité (roman réorganisé a posteriori vs roman à bifurcations borgésiennes), et, corrélativement, d'après la relation qu'elles entretiennent avec le roman papier : Twitter peut constituer un modèle alternatif aux expérimentations de livres à plusieurs voix menées sur le Web depuis plus d'une décennie aussi bien qu'un simple outil de diffusion de l'œuvre auctoriale maîtrisée et fermée.

5 septembre 2009, http://www.actualitte.com/actualite/13229-premier-roman-ecrit-Twittercinema.htm, consulté le 19 avril 2010.

18. http://twiller.tcrouzet.com/.

19. http://buboneka.blogspot.com.

20. http://culturebox.france3.fr/all/15520/Avec-Thomas-Drimm\%2C-Didier-van-Cauwelaertcree-le-1 er-roman-feuilleton-sur-telephone-mobile\#/all/15520/Avec-Thomas-Drimm,-Didiervan-Cauwelaert-cree-le-1er-roman-feuilleton-sur-telephone-mobile/.

21. François Gayrard, « Le Roman d'Arnaud », Numerikbook's blog, l'actualité de l'édition numérique, http://numerikbook.wordpress.com/le-roman-darnaud/. Voir aussi la synthèse d'Hervé Bienvault, «Les nouveaux romans "feuilletons" », Aldus, 8 octobre 2009, http:// aldus2006.typepad.fr/mon_weblog/2009/10/les-nouveaux-romans-feuilletons.html, consulté le 19 avril 2010. 


\section{Une littérature sans contrainte}

L'une des originalités les plus profondes du Twaïku ne tenait ni à sa forme ni à son médium, mais à la possibilité offerte par ce que l'on appelle les hashtags ${ }^{22}$ de rechercher et d'identifier des poèmes produits n'importe où dans le réseau sans connaissance préalable de leur auteur et, en quelque sorte, de pouvoir constituer des constellations et des recueils poétiques totalement disjoints, à rebours des modes d'auctorialité convenus. En faisant jouer à plein la sérendipité, Twitter autorise la production de dispositifs accumulatifs contrôlés (comme le projet de créer le plus long poème du monde sur Twitter ${ }^{23}$, poème qui comporte, à la date où cet article est écrit, plus de 520000 vers) ou libres, par croisements a posteriori et invention d'une totalisation de circonstance.

Or le Web 2.0 conduit ces évolutions fondamentales que sont la collectivisation des contenus (permise par ce que l'on appelle le cloud computing) et la socialisation de l'universel reportage jusqu'à un point plus troublant encore : la littérature y acquiert la capacité de sortir des espaces pensés pour l'expression littéraire, de s'installer, sous forme de jeux de mots, de plaisanterie, de dialogues théâtralisés, de poèmes, ou d'une expression dénudée de la souffrance, à l'intérieur des dialogues sociaux. Le Web de seconde génération est un web du flux, un web sans web, où même le support numérique s'est dissous ${ }^{24}$, sphère dialogique en mouvement où la littérature s'instille dans la dynamique des liens. Resocialisation de l'expression poétique et du fait littéraire en général autant que poétisation des relations sociales, la littérature renaît avec la conversion textuelle de notre relation induite par leur basculement dans cet empire du texte qu'est le Web. Qu'elle emprunte la puissance des mécanismes dialogiques sur Twitter ou les possibilités de critique par « commentaires » à valeur métatextuelle ou intertextuelle sur Facebook, la littérature renaît de notre besoin d'enrichir et de spiritualiser les échanges en investissant un espace où tous les déplacements esthétiques, tous les jeux textuels et les inserts poétiques sont possibles. Technique de manipulation symbolique des états, de transformation formelle des sentiments et des idées, de la production de mondes possibles à partir des lacunes du réel, l'écriture trouve dans l'accès que lui offrent les réseaux sociaux à notre intimité et à nos affects un espace privilégié où se réaffirment et sa puissance et son universalité. Devoir passer par l'écrit pour transmettre un événement, un lieu, un état d'âme, médiatiser son existence, ses projets ou amours, dans un espace scriptural public, rendre ses humeurs et ses émotions amplifiables ou « commentables » par d'autres

22. Balises thématiques utilisées par la communauté des utilisateurs de Twitter pour regrouper des tweets autour d'un sujet, d'un lieu ou d'une personne et notées par le signe dièse «\#». 23. http://www.longestpoemintheworld.com.

24. Voir par exemple les analyses de Nova Spivak: «Welcome to the stream : the next phaseof the Web », Twine, 8 mars 2009, http://www.twine.com/item/128lryv9z-46/is-thestream-is-the-next-new-metaphor commenté par Thierry Crouzet : «Vers un web sans site web », Le peuple des connecteurs, 17 août 2009, http://blog.tcrouzet.com/2009/08/17/versun-web-sans-site-web, consulté le 19 avril 2010. 
textes, conduit à réaffirmer comme jamais la séduction triomphante, l'efficace, la disponibilité infinie du style et de la fiction. Loin d'être l'empire du trivial et de l'éphémère, cette littérature généralisée joue autant des pouvoirs d'amplification (ce que l'on pourrait nommer une microrhétorique), que de ceux de la dissimulation ou de l'ombrage : elle amplifie ou au contraire voile, les statuts et les tweets tour à tour avouent et dissimulent, confessent et commentent, cryptent et décodent, précisent et développent, basculent dans la fiction ou exhument les références, s'attachent au temps long, à la mémoire, fixent des états à valeur paradigmatique ou scandent les passages.

À défaut de produire une stylistique générale du microblogging encore à venir, je prendrai ici quelques exemples de fil de discussion issus de Twitter et de Facebook - par respect du caractère privé de certaines discussions, les noms ont été remplacés par des initiales; on notera par ailleurs que les extraits cités ici ne restituent que très partiellement la richesse dialogique des discussions en n'incluant ni les commentaires, ni les remarques d'appréciation, ni les médias, extraits vidéo ou audio, qui en constituent souvent la sève - pour ces mêmes raisons, comme pour des impératifs techniques, l'adresse bibliographique de ces travaux ne saurait être donnée. Ici, par exemple, tel écrivain (proche de la revue Inculte) ironise sur l'existence :

ML constate que l'atelier des affects improbables n'est pas un open-space avant que de proposer une brève épiphanie poétique :

ML s'abritant sous une ombrelle mauve du soleil qui passe

Tel blogueur et poète joue des métalepses :

IS couché sur le papier

Telle blogueuse réputée accumule les jeux de mots et les formules énigmatiques :

LL sabre et muse en Aragonne.

$[\ldots]$

LL Sous aucun prétexte.

Tel autre écrivain, dans l'orbite de Léo Scheer, profite de Facebook pour produire des instantanés :

ET Tout le corps tendu que cingle la neige se raidit et tend vers la tâche. Et certes, l'on voudrait mourir.ET Elle redescend l'escalier, tenant la rampe, sans toutefois vous quitter du regard et, comme l'on fait un pas pour la suivre, Mimose retire l'escalier et vous sourit à son tour, bras croisés comme devant, en armes, au bord de la falaise que lèche une mer grise.

Cette verve formulaire n'est ni propre à des blogueurs consacrés ni à des écrivains déclarés, car innombrables sont les « fils » où les vies ordinaires acquièrent une densité inédite par la récriture, ainsi d'une autre chronique en ligne célèbre, tenue par une jeune universitaire : 
AA en est au stade où on oublie qu'on oublie

AA a passé la nuit la plus féline du monde et va peut-être se recoucher tandis que les fauves mangent, les champs brûlent, etc.

AA célèbre un mariage improbable et illimité

Cette intensification scripturale des mots intérieurs et cette transmutation en énigmes et symboles des realia ordinaires viennent couronner la longue ascension du quotidien comme objet digne de la littérarité ${ }^{25}$; elle touche à la littérature par l'ouverture faite à l'interprétation et à la glose, comme par un possible réemploi dans notre propre expérience, les bénéfices heuristiques et cognitifs de la mise en partage des sentiments et réflexions de celui qui les expose ne lui étant pas limités. Les commentaires (sur Facebook) et autres reprises sous la forme de citation (les « retweet » de Twitter, c'est-à-dire la redistribution virale d'un message) prennent une place essentielle : loin de se limiter à un rôle de médiation entre l'espace du privé (opaque) et l'espace public (transparent), ils peuvent réinterpréter, surinterprétrer, mésinterpréter, contre-interpréter, déplacer la parole initiale, à la manière des brillantes conversations de salon du XVII siècle. Prenons quelques exemples, d'abord du côté du plaisir propre à l'esprit d'escalier :

$M C$ va aller dévaster quelques livres qui l'ont bien cherché.

CL > Il faudrait dévaster Flatland, Flatland l'a bien cherché. Et après le Grabinoulor. Et après John Kennedy Toole. Et après Nabokov.

$[\ldots]$

MC demande des souvenirs à la pluie.

$>$ DD ... et les nuages de les distiller au goutte à goutte!

$>$ IV bien vu puisque l'eau tonne;-)

$[\ldots]$

MC Repasse et manque.

NT $>$... le pli.

NT $>$ de fer?

ST $>$ il est repassé et nous manque terriblement...

Ailleurs, tel écrivain plaisante :

ME rêverait de s'appeler Ödön von Horváth.

SD Et d'aller voir Fantasia au cinéma.

ME Je ne vais jamais aux Champs-Élysées, à part pour acheter un billet d'avion chez Iran Air, ce que je me garde bien de faire. Donc aucun problème, je suis immortel.

L'attestation référentielle se mêle à l'exploration métaphorique :

OR et nous ne tomberons jamais seuls

$>\mathrm{JJ}$ voir beckett in « Tous ceux qui tombent », petit film rare, une perle...

$>\mathrm{KM}$ « réussir à chuter vers le haut»

25. Voir sur ce sujet Michael Sheringham, Everyday Life. Theories and Practices from Surrealism to the Present, Oxford, Oxford University Press, 2006. 
Le jeu de miroitement de l'énigme et de l'allusion - dont le destinataire réel peut rester un observateur extérieur et le secret aussi profondément enfoui que celui des sonnets de Shakespeare-, les pièges interprétatifs et leurs nécessaires mésinterprétations, le jeu parfois énigmatique des pseudonymes lorsque des entités abstraites ou des écrivains du passé prennent la parole, sont la matrice d'une poétisation générale de l'expérience que son ouverture sémantique et métaphorique rapproche de ce que nous avions l'habitude d'appeler « littérature ».

Plusieurs projets novateurs d'ateliers littéraires numériques émergents (aux éditions Léo Scheer ou en ligne sur Publie.net) et nombre des écrivains les plus brillants de leur génération (Éric Chevillard, Emmanuel Tugny, Matthieu Larnaudie, Olivier Rohe, etc.) s'approprient le microblogging, au moment même où des communautés littéraires non professionnelles comme celle polarisée par Remue.net et le projet Liminaire de Pierre Ménard ${ }^{26}$ entament simultanément l'hybridation de l'écriture numérique et des ateliers d'écriture traditionnels et un travail de réflexion sur leurs pratiques - auquel cet article doit beaucoup, qu'il s'agisse de reconnaître et de pérenniser certaines pages poétiques issues des flux ${ }^{27}$ ou de penser le statut référentiel des tweets, comme dans l'échange que j'ai mené pour ce travail avec la communauté remue.net :

Quelle différence entre @ remuenet @agefen Twitt litt c'est effort au style, dans l'élégance ou la rupture sinon lire, écrire, rêver, dormir, tout ça se fait en 140.

Aucune, évidemment, nulle différence entre la vie, le net, l'écriture, la lecture, la littérature sinon que soleil brille dehors 140

Ou avec François Bon, qui insiste sur la novation radicale constituée par ces écritures en flux du corps et de l'âme mêlés off world:

fbon@agefen 1/2 « toute littérature est assaut contre la frontière » Franz Kafka, je pensais à toi : le microblogging devient forme littéraire

fbon@agefen 2/2 quand le blog ou le twit ou le FB s'oriente intentionnellement vers la frontière, et non quand il mime roman ou pose à l'auteur

Nous sommes ici dans un territoire inédit, bien plus déconcertant pour notre culture lectoriale et nos axiologies que celui des simples fictions hypertextes ${ }^{28}$ qui relèvent, me semble-t-il, d'œuvres faussement ouvertes et de la continuation d'une forme de poétique de la nécessité cachée derrière de superficielles innovations. Tenter de rapporter au modèle ancien du livre ${ }^{29} \mathrm{ces}$

26. http://www.liminaire.fr.

27. Voir par exemple : http://remue.net/spip.php?article3394.

28. Voir en anglais : http://www.eastgate.com/malloy/ ou http://www.adrienneeisen.com; en français les entreprises de S. Bouchardon (http://www.les12travaux.com), de J.-P. Balpe (http://jpbalpe.blogdrive.com/) ou de X. Malbreil (http://www.livresdesmorts.com).

29. On pourra consulter néanmoins la belle expérience de « Tumulte» comme celle d'Année compte double, tentative de mise en forme des contributions au petit journal de « Tiers Livre », du $1^{\text {er }}$ décembre 2007 au 30 novembre 2008, « augmentées de leur index alphabétique, catégorique et subjectif » par Martine Sonnet : http://www.calameo.com/books/000098947b2666fa59be3. 
« fils » de discussion où les acteurs remplacent le support, où le flux même est le contenu, la dynamique la poésie, où commentaire et lecture, compréhension et récriture s'indifférencient, est sans doute infiniment limitatif. Ce sont plutôt les cartes de visite cornées de Mallarmé et ses « éventails », les envois et exlibris de Larbaud, les bons mots des moralistes classiques, les gloses infinies de Montaigne, les rhizomes de Deleuze et Guattari qu'il faudrait invoquer ${ }^{30}$.

Les lignes de force et les dispositifs de Tumulte pointent du doigt, par défaut, l'ordinaire des couplets, rengaines et complaintes, sur la fin et/ou le retour du roman. En refusant de simuler une continuité narrative, en maintenant la distinction entre figuration et fiction de soi et des autres, en faisant coexister temps de la réalité, de l'écriture et de la narration, celui-ci, par l'acuité et la célérité avec lesquelles sans cesse il expérimente l'épaisseur du monde et la matière de la langue, approche au plus près du point de contact entre soi et le réel, jusqu'à l'acte littéraire ${ }^{31}$.

suggère François Bon à partir de l'expérience de Tumulte. Abandonnant en chemin la sacralité intransitive de la parole et les structures formelles propres à la littérature industrielle pour retrouver des pratiques de dialogue communautaire et d'invention collective plus anciennes et réinscrites dans le continuum des échanges sociaux et de la vie morale individuelle, le microblogging nous plonge dans un univers de flux où l'écrit est en attente d'échos et la vie relationnelle en demande de parole, que l'on y voie ou non, comme le suggère Nicolas Gary, l'avenir de l'écriture ${ }^{32}$. Et dans cette conversion numérique de l'expérience humaine ${ }^{33}$, c'est peut-être à une extension immense du champ de la littérature et à un réinvestissement complet d'un exercice esthétique supposé misanthrope que nous assistons.

Tirer les conséquences sur notre théorie de l'œuvre et de l'auteur de la dimension interactive et communicationnelle du microblogging littéraire, renoncer à opposer média, normes techniques et contenus, intention et expression, identité singulière et médiation collective, telles sont, quoi qu'il en soit, les conditions de nécessité pour penser l'intérêt esthétique de cette

30. La «banque de concepts» du projet de la « Molecular Twitter Party» se propose de « réfléchir aux croisements dans le monde digital entre les concepts Deleuzo-Guattariens et la machine Twitter » conçue comme « un dispositif qui segmente nos vies quotidiennes, ordinaires, de/re/codant des lignes de segmentation moléculaires » (http://moleculartwitterparty.blogspot.com/2009/08/conference-proposal.html, je traduis).

31. Dominique Dussidour, " "Les livres nous accompagnent même dans nos chutes" ", remue. net, 2 décembre 2006, http://remue.net/spip.php?article1987, consulté le 19 avril 2010.

32. Interview de Nicolas Gary dans Books, n 7, juillet-août 2009, http://www.booksmag. $\mathrm{fr} /$ magazine/c/nicolas-gary-le-blog-est-depasse-aujourd-hui-on-ecrit-des-romans-sur-twitter1.html, consulté le 19 avril 210.

33. Sur le thème de la conversion numérique on consultera l'essai, intéressant mais embryonnaire, de Milad Doueihi, La Grande Conversion numérique, Paris, Seuil, 2008, essai que l'on enrichira ne serait-ce que par quelques exemples issus des pratiques sociales: http://mashable.com/2009/04/10/extraordinary-twitter-updates. 
pratique et en comprendre les modes de généricité. C'est alors renverser certains des traits définitoires traditionnels de la littérature (comme acte de création individuel indifférent à son support et intentionnellement destiné à être publié et lu de manière universelle, définition dont on sait au demeurant les difficultés à caractériser comme littéraire L'Iliade et L'Odyssée, les Vies de saints médiévales, les Pensées de Pascal, les romans baroques mondains, les Mémoires historiques de Saint-Simon, la Correspondance de Flaubert, etc.) au profit d'une vision qui fait de l'œuvre un dispositif sémiotique original, un artefact, dirait Roger Pouivet dont nous suivons mutatis mutandis ici les propositions ${ }^{34}$, un objet textuel profondément inscrit dans un contexte historique, dépendant d'un croisement d'intentions parfois imprévisibles et en négociation constante avec ses supports.

Tirer les conséquences esthétiques de notre immersion dans les pratiques d'écriture numérique et observer les premières générations d'écrivains nés à Internet peut nous conduire à penser en terme d'émergence, d'innovation formelle, de contamination, d'influence indirecte, de déplacement. Que l'amont de la création (l'information existentielle), le processus de celle-ci, ou son aval, les espaces de diffusion et usages, soient affectés par la conversion numérique, nous conduit certes à historiciser le fait littéraire, mais aussi à en reconnaître, dans cette esthétisation du particulier et cette ostentation de soi ${ }^{35}$ qui sont peut-être des traits déterminants de la modernité, les facteurs de continuité. Car le Web 2.0, dont les caractères sont bien connus (interactivité accrue, personnalisation d'accès, transversalité communautaire, sémantisation croissante, diffusion dans les objets) et rapprochent paradoxalement, on l'a vu, l'écriture virtuelle de nos usages conversationnels et de nos échanges affectifs communs, aligne le Web sur le quotidien et en diminue la rugosité technologique : la pénétration de plus en plus profonde, de plus en plus ubiquitaire du numérique, qui nous escorte dans nos pratiques les plus ordinaires et les plus intimes de lecture et de rédaction, s'accompagne inversement d'une humanisation et d'une socialisation croissante des techniques. Ici, le virtuel numérique poursuit le vieux fantasme d'abstraction et de dématérialisation propre à la fiction, à la fois anneau de Gygès, dispositif d'accès à la conscience d'autrui, production d'une réalité augmentée ou d'une réalité réparée, mémoire communautaire et machine à produire récritures, liens et analogies, arbre textuel immense nous conduisant hors le monde, tout en supportant notre désir de possibles.

Alexandre Gefen

Université de Bordeaux - TELEM

34. Voir Roger Pouivet, Qu'est-ce qu'une ouvre d'art?, Paris, Vrin, 2007, p. 63-64.

35. Voir par exemple sur ce sujet l'article de Jill Walker, « Mirrors and shadows : the digital aestheticisation of oneself $»$, https://bora.uib.no/bitstream/1956/1136/1/mirrorsandshadowsfinal.pdf, consulté le 19 avril 2010. 